\title{
Strategies for Prevention of Deafness in India
}

Rabindran $^{1^{*}}$, Shasidaran $^{2}$

${ }^{1}$ Consultant, Neonatologist, Billroth Hospital, Chennai, India

${ }^{2}$ Senior Resident, Department of Radiology, S.R.M. Medical College and Research Centre, Chennai, India

DOI: $10.36347 /$ sjams.2020.v08i09.022

| Received: 09.09.2020 | Accepted: 17.09.2020 | Published: 19.09.2020

*Corresponding author: Rabindran

\section{Abstract}

Deafness is complete inability to hear whereas Hearing impairment is complete or partial inability to hear. There are 4 grades of hearing loss based on hearing threshold of the better ear. In India, nearly $6.3 \%$ of the population have hearing impairment and the prevalence of childhood-onset deafness is $2 \%$. The etiological factors leading to deafness are manifold. There is a rise in hearing loss secondary to noise exposure. Severe infections, meningitis, some medications cause hearing impairment. Maternal TORCH infection and chronic ear infection also contribute to hearing loss. It is estimated that nearly $50 \%$ of hearing loss are preventable. The 3 strategies are Primary prevention, Secondary Prevention and Tertiary prevention. Primary preventive strategies of deafness include education, hearing conservation programmes and legislation. Immunization of mothers against rubella, Immunization of children against H. influenza, S. pneumonia, measles and mumps can reduce childhood deafness. WHO advises that children should limit exposure to loud sounds? Improved maternal and child health practices would prevent hearing loss. Newborn and infant hearing screening should be mandatory. Awareness against excessive noise exposure should be created. Secondary preventive strategies of deafness include environmental modification. For children admitted to special schools or rehabilitation centres an audiological evaluation should be done prior to admission. There should be regular School-based hearing screening. Tertiary preventive strategies of deafness include hearing aids and rehabilitation. Rehabilitation measures should be provided for those who have developed hearing loss. Early detection and prompt management is the key to address the problem of deafness in India.

Keywords: Deafness, Primary Prevention, Hearing Aid.

Copyright @ 2020: This is an open-access article distributed under the terms of the Creative Commons Attribution license which permits unrestricted use, distribution, and reproduction in any medium for non-commercial use (NonCommercial, or CC-BY-NC) provided the original author and source are credited.

\section{INTRODUCTION}

According to World Health Organisation (WHO), Deafness is defined as complete inability to hear from one or both ears. This is considered equivalent to profound hearing impairment, i.e., hearing loss greater than $80 \mathrm{~dB}$ averaged at multiple frequencies of $0.5,1,2,4 \mathrm{kHz}$. The term Hearing impairment is used to mean complete or partial inability to hear from one or both ears. This is equivalent to mild hearing impairment, i.e., hearing loss greater than $25 \mathrm{~dB}$ averaged at multiple frequencies [1]. There is another term called disabling hearing impairment which means moderate or worse hearing impairment in the better ear. This corresponds to hearing threshold level for the better ear of greater than $40 \mathrm{~dB}$ (for age $>14$ years) and greater than $30 \mathrm{~dB}$ (for age $<15$ years) averaged at multiple frequencies. There are 4 grades of hearing loss based on hearing threshold of the better ear; Slight / Mild (26-40 dB), Moderate (41-60 dB), Severe (61-80 $\mathrm{dB})$ and Profound $(>80 \mathrm{~dB})[2]$.

\section{Problem Statement}

Deafness is a concerning issue nowadays because as per WHO statement in March 2020, approximately 466 million people worldwide (which is equivalent to $5 \%$ of global population) have disabling hearing loss [3]. Out of this number nearly 34 million are children. More worrisome is the projected estimate for 2050 that more than 900 million people globally (which approximates to 1 in every 10 people) is expected to have disabling hearing loss. WHO in 2018 stated that in India, nearly 63 million people $(6.3 \%$ of the population) have hearing impairment? Out of which the prevalence of adult-onset deafness is $7.6 \%$ and childhood-onset deafness is $2 \%$ [4]. Due to the growing problem of hearing impairment, the National Programme for Prevention and Control of Deafness (NPPCD) was established. Its primary aim is early identification, diagnosis and treatment of ear problems responsible for hearing loss. 


\section{Etiological Factors}

Hearing loss may be adult onset or childhood defect. The risk factors and etiological factors leading to deafness are manifold [1]. With advancement of genetic diagnosis, many idiopathic hearing defects are being attributed to genetic disorders nowadays. Nearly 75$80 \%$ of hearing loss secondary to genetic etiology are inherited by recessive genes, $20-25 \%$ are inherited by dominant genes, 1-2\% are inherited by X-linked pattern, and fewer than $1 \%$ are inherited by mitochondrial inheritance [5]. Due to modern life-style and excessive exposure to gadgets there is a rise in hearing loss secondary to noise exposure [6]. Though due to the advancement in medical therapeutics, the infections are being controlled with broad-spectrum antibiotics, there is emergence of resistant microorganisms and superbugs leading to severe infections and meningitis ultimately contributing to infection induced hearing impairment [2]. Moreover some of the medications and antibiotics, anti-tubercular drugs have hearing impairment as a significant side effect [7].

Due to advancement of neonatal care more number of extreme preterms are surviving nowadays and they are at higher risk of developing hearing impairment apart from birth complications and hyperbilirubinemia induced hearing impairment in neonates. Significant trauma to ear may also lead to deafness [8]. Maternal TORCH infections particularly cytomegalovirus, syphilis and rubella, can also lead to hearing loss [9]. Moreover any chronic ear infection may ultimately end in hearing impairment. Practice of Consanguineous marriage, use of antimalarial drugs, and irrational use of aminoglycosides, Iodine deficiency and illiteracy are risk factors for Sensory Neural Hearing Loss. Inadequate health care services particularly in rural areas, poor health education, inadequate primary ear care programme, poor environmental sanitation and neglect of upper respiratory infection contribute to conductive hearing loss.

\section{Prevention}

Hearing loss can be prevented. It is estimated that nearly $50 \%$ of cases of hearing loss are preventable [10]. Nearly $60 \%$ of childhood hearing loss in children can be prevented [1]. Like any preventable disorder there are 3 types of strategies for prevention of deafness. The 3 strategies are Primary prevention, Secondary Prevention and Tertiary prevention. Primary prevention is an intervention implemented before there is evidence of injury. The intent is to decrease and eliminate causative risk factors (risk reduction). Primary preventive strategies of deafness include education, hearing conservation programmes and legislation. Secondary Prevention is an intervention implemented after the disease has begun, but before it is symptomatic. The intent is early identification (through screening) and treatment. Secondary preventive strategies of deafness include environmental modification. Tertiary prevention is an intervention implemented after the disease is established. The intent is to prevent severe sequelae. Tertiary preventive strategies of deafness include hearing aids and rehabilitation. Tertiary prevention aims to reduce disability, delay complications, and restore functions.

\section{Indian Initiative}

Taking the growing problem of deafness into concern, National Program for Prevention and Control of Deafness (NPPCD) was started in India in the year 2006. The aim is to prevent deafness using all 3 strategies namely primary, secondary and tertiary ear care. The program concentrates on preventing hearing loss secondary to diseases and trauma. Early identification and prompt treatment of ear disorders are given importance. Medical rehabilitation is provided to persons with hearing impairment. Innovative and effective information, education and communication strategies are used to increase public awareness about preventable deafness. Due to large population and huge burden of hearing impairment in India, NPPCD has been integrated with the National Health Mission at state and districts levels.

\section{Primary Preventive Strategies for Prevention of Deafness}

Primary prevention is an intervention implemented before there is evidence of injury. The intent is to decrease and eliminate causative risk factors (risk reduction). Primary preventive strategies of deafness include education, hearing conservation programmes and legislation.

\section{Hearing Conservation Programmes}

Since majority of childhood onset hearing loss are caused by infections of mother antenatal or to children postnatally, vaccination remains as a cornerstone to prevent such hearing loss. Immunization of adolescent girls or expectant mothers against rubella can prevent congenital rubella syndrome. Immunization against $H$. influenza, S. pneumoniae decreases infectious meningitis associated hearing loss. Nearly $30 \%$ of childhood hearing loss is caused by diseases such as measles, mumps, and rubella, meningitis and ear infections. Immunization against measles and mumps can also reduce childhood deafness [11]. Early management of otological problems by providing printed handouts on primary and secondary ear care, avoidance of ototoxic drugs and advice on hazards of consanguineous marriage through counselling are strategies of primary prevention of deafness.

\section{Training}

Primary-level physicians and health care workers particularly in remote regions and rural areas should be trained about the importance of early intervention, available treatment options and relevance of good ear examination for identification of ear 
diseases early. Facilities for services should be easily accessible to the people. Referral of patients with hearing loss when required to tertiary centres should be practised appropriately.

\section{Education}

Lack of Education and awareness about the disorder is the main roadblock in any preventable disease. There are various programs targeting certain groups like school-age children, adolescents and workers [12]. WHO advises that children and adolescents should limit exposure to loud sounds? Personal audio players should be restricted to about an hour a day. In situations where noise exposure cannot be decreased, use of antioxidants is being suggested to prevent noise -induced hearing loss [13].

\section{Improved Maternal and Child Health Practices}

Prolonging labour and preventing premature delivery through good antenatal care can reduce prematurity associated hearing impairment. Around $17 \%$ of childhood hearing loss results from complications at birth, including prematurity, low birth weight, birth asphyxia and neonatal jaundice. Improved maternal and child health practices would help to prevent these complications [11]. Use of ototoxic medicines in pregnant women and children is responsible for $4 \%$ of childhood hearing loss and it should be discouraged [11]. Improved maternal nutrition, awareness of hygienic practices, promotion of safe birth, prompt management of neonatal infections and jaundice help to improve Maternal and neonatal care. Newborn and infant hearing screening should be made mandatory and tracking by local health workers should be done [14]. Congenital or early- onset hearing loss should be identified early and treatment should be started early since hearing is mandatory for proper speech and language development among children.

\section{Raising Awareness}

Awareness against excessive noise exposure should be created. This awareness will increase the practice of using hearing protectors. Awareness about healthy ear care practices like avoiding insertion or instillation of any substance to reduce ear infections is necessary. Parents and caregivers should be advised to refrain from using home remedies for children with ear pain. They must be aware to consult with a medical practitioner immediately to prevent chronic ear infections and subsequent hearing loss. Children should be aware about the dangers of loud sounds especially in a recreational context [15]. This awareness will lead to behaviour modification and promote safe listening. This practice change can prevent noise-induced hearing loss during childhood and adolescence. Awareness to the public is necessary to reduce the stigma associated with hearing loss, hearing devices and alternative communication methods.

\section{Legislation, Regulation and Monitoring}

Strict rules and laws are mandatory particularly to reduce occupational hazard associated deafness. Noise limits should be strictly followed in workplaces and violations should be strictly penalised. Legislation should be formulated regarding restriction of sale and ototoxic medicines. Indiscriminate and irrational use of such medications should be regulated and monitored. If used periodic audiological monitoring should be done to identify hearing loss at an early stage. Health care professionals should be sensitized regarding the importance of conserving hearing in people taking these medications. A specific World Hearing Day is promoted as a yearly event to raise public awareness in preventing hearing impairment. Noise levels in the community should be regulated and monitored, especially at recreational venues and sports arenas. Due to technological advancements, there are safety features which can be enabled to personal audio systems which a reduce the risk of hearing loss. Organizations of people with hearing loss, parents and family support groups should be strengthened.

\section{Secondary Preventive Strategies for Prevention of Deafness}

Secondary Prevention is an intervention implemented after the disease has begun, but before it is symptomatic. The intent is early identification (through screening) and treatment. Secondary preventive strategies of deafness include environmental modification.

\section{Change of Environment - Workplace Noise Regulation}

Noise is now well known as an occupational hazard [16]. The National Institute for Occupational Safety and Health proposed that majority of hearing loss is not due to age, but due to noise exposure [17]. There is a hierarchy of hazard control which specifies the different levels of controls to reduce noise exposure and prevent hearing loss. They provide standards for engineering controls and personal protective equipment. Industries should make a policy of providing personal hearing protector devices [18]. There are many types of hearing protectors available. Some will block out all noise, whereas some allow certain noises to be heard. Therefore personal hearing protector devices should be tailored to both the worker and type of employment. Good ear care and general hygiene can help to prevent ear infections and glue ear. They can be treated by prompt medical and surgical interventions.

\section{Implementation of Screening and Intervention Programmes \\ For children admitted to special schools or} rehabilitation center an audiological evaluation should be done prior to admission even though the child may have other disorder or disability as the primary pathology. Similarly for children with learning difficulties, Audiological evaluation should be done 
before proceeding to further evaluation, since hearing loss may be the reason for poor academic performance. There should be regular School-based hearing screening. The purpose of such an endeavour is to identify common ear diseases early, refer to appropriate centre and through early intervention prevent the complications and hearing loss.

\section{Tertiary Preventive Strategies for Prevention of Deafness}

Tertiary prevention is an intervention implemented after the disease is established. The intent is to prevent severe sequelae. Tertiary preventive strategies of deafness include hearing aids and rehabilitation. Tertiary prevention aims to reduce disability, delay complications, and restore functions.

\section{Making Appropriate Technologies Accessible}

Even if there is onset of hearing loss, tertiary preventive measures help in preventing sequelae. First thing is to remove the stigma about using hearing aids [19]. Now there are newer cosmetically better hearing aids available in the market [20].These devices should be made easily accessible and at an affordable cost. Recent advances in the field of hearing aids and cochlear implants have improved the quality of hearing aids for usage over all age groups. However there is need for ongoing support and motivation for people using these devices.

Rehabilitation measures should be provided for those who have developed hearing loss. Communication and education should be accessible. Since hearing is vital for proper speech and language development, a deaf child may get more benefit from early introduction to language. Such rehabilitative measures can be provided in the form of verbal communication (auditory-verbal and auditory-oral therapy) [21]. There are other alternative means of communication, like sign language, total communication, bilingual / bicultural (bi-bi) teaching, cued speech and lip reading approaches [1]. Use of loop and FM systems in classrooms and public places, provision of captions on audiovisual media help to provide accessibility of communication for people with hearing loss. Such measures can reduce adverse impact of hearing loss.

\section{CONCLUSION}

Management and prevention of deafness needs a multidisciplinary approach. People like medical experts, experts from basic and hearing sciences, and technology should work together to provide strategies that could prevent hearing impairment and its sequelae. Early detection and prompt management is the key to address the problem of deafness in India.

\section{REFERENCE}

1. Deafness and hearing loss Fact sheet.World Health Organisation. №300". March 2015.

2. Elzouki AY, Harfi HA, Nazer H, Oh W, Stapleton FB, Whitley RJ. Textbook of clinical pediatrics. Springer Science \& Business Media; 2011 Oct 29.

3. Olusanya BO, Neumann KJ, Saunders JE. The global burden of disabling hearing impairment: a call to action. Bulletin of the World Health Organization. 2014 Feb 18;92:367-73.

4. Davey S, Maheshwari C, Raghav SK, Singh N, Muzammil K, Pandey P. Impact of Indian public health standards for rural health care facilities on national programme for control of deafness in India: The results of a cohort study. Journal of Family Medicine and Primary Care. 2018 Jul;7(4):780.

5. Rehm HL. Understanding the genetics of deafness: A guide for patients and families. Harvard Medical School Center for Hereditary Deafness; 2003.

6. World Health Organization, Smikey L. 1.1 billion people at risk of hearing loss. World Health Organization: Geneva, Switzerland. 2015.

7. Curhan SG, Shargorodsky J, Eavey R, Curhan GC. Analgesic use and the risk of hearing loss in women. American journal of epidemiology. 2012 Sep 15;176(6):544-54.

8. Eggermont JJ. Acquired hearing loss and brain plasticity. Hearing Research. 2017 Jan 1;343:17690.

9. Fowler KB. Congenital cytomegalovirus infection: audiologic outcome. Clinical infectious diseases. 2013 Dec 15;57(suppl_4):S182-4.

10. Graham J, Baguley D, editors. Ballantyne's Deafness. John Wiley \& Sons; 2009 Jul 6.

11. World Health Organization. Childhood hearing loss: Act now, here's how. World Health Organization; 2016.

12. Davis A, McMahon CM, Pichora-Fuller KM, Russ S, Lin F, Olusanya BO, Chadha S, Tremblay KL. Aging and hearing health: the life-course approach. The Gerontologist. 2016 Apr 1;56(Suppl_2):S25667.

13. Stucken EZ, Hong RS. Noise-induced hearing loss: an occupational medicine perspective. Current opinion in otolaryngology \& head and neck surgery. 2014 Oct 1;22(5):388-93.

14. Lasak JM, Allen P, McVay T, Lewis D. Hearing loss: diagnosis and management. Primary Care: Clinics in Office Practice. 2014 Mar 1;41(1):19-31.

15. de Laat JA, van Deelen L, Wiefferink K. Hearing screening and prevention of hearing loss in Adolescents. Journal of Adolescent Health. 2016 Sep 1;59(3):243-5.

16. Oishi N, Schacht J. Emerging treatments for noiseinduced hearing loss. Expert opinion on emerging drugs. 2011 Jun 1;16(2):235-45.

17. "CDC - NIOSH Science Blog - A Story of Impact..." cdc.gov. 
18. El Dib RP, Mathew JL, Martins RH. Interventions to promote the wearing of hearing protection. Cochrane database of systematic reviews. 2012(4).

19. Sparrow R. Defending deaf culture: The case of cochlear implants. Journal of Political Philosophy. 2005 Jun;13(2):135-52.
20. Keidser G, Convery E. Self-fitting hearing aids: Status quo and future predictions. Trends in Hearing. 2016 Apr 9;20:2331216516643284.

21. Schilder AG, Chong LY, Ftouh S, Burton MJ. Bilateral versus unilateral hearing aids for bilateral hearing impairment in adults. Cochrane Database of Systematic Reviews. 2017(12). 